\title{
TILINGS WITH CONGRUENT TILES
}

\author{
BY BRANKO GRÜNBAUM ${ }^{1}$ AND G. C. SHEPHARD
}

Introduction. The purpose of this paper is to survey recent results related to the second part of Hilbert's eighteenth problem (see Hilbert [1900]). This problem, which is concerned with tilings of Euclidean space by congruent polyhedra, will be stated below after the necessary terminology has been introduced. Although Hilbert's original question was answered by one of his assistants (Karl Reinhardt) more than fifty years ago, there remain many unsolved problems in this area of mathematics; a description of recent results therefore seems to us to be of considerable interest.

Three surveys of developments related to Hilbert's problems (Aleksandrov [1969], Browder [1976], Kaplansky [1977]) have been published in recent years, but they contain no mention of the remarkable advances made during the last decades in connection with the problem that concerns us. An even more cogent reason for publishing this survey is that much of the material results from the work of crystallographers, and no mention of it appears in the recognized mathematical literature. Reasons for this disregard are easy to find. Current fashions in mathematics applaud abstraction for its own sake, regarding it as the highest intellectual activity-whether or not it is, in any sense, useful or related to other endeavors. Mathematicians frequently regard it as demeaning to work on problems related to "elementary geometry" in Euclidean space of two or three dimensions. In fact, we believe that many are unable, both by inclination and training, to make meaningful contributions to this more "concrete" type of mathematics; yet it is precisely these and similar considerations that include the results and techniques needed by workers in other disciplines. Moreover, throughout the history of mathematics, fundamental ideas for other branches of mathematics have been motivated by "intuitive" geometric questions; irrational numbers, calculus, axiomatics and topology are only a few of the most obvious "inventions" that originated in this manner. It seems to us to be foolish and presumptuous to believe that ours is the first generation which needs no more the inspiration that can be found in studying simple geometric objects and their mutual relations. By showing that there are still many open and difficult (yet interesting and easily understood) problems, we hope to persuade some readers that this is an area of mathematics worthy of their attention.

Received by the editors April 15, 1980.

1980 Mathematics Subject Classification. Primary 51M20, 52A45; Secondary 05B45, 10E30, 52A15, 52A25, 52A43.

${ }^{1}$ Research supported in part by the National Science Foundation Grant No. MCS7701629A01. 
In this connection we strongly approve of the recent policy of Mathematical Reviews to include papers from crystallographic journals. By ignoring this material in the past several decades, the mathematical community has suffered a great loss of information.

Background. There exists a vast literature on the subject of tiling, and almost every imaginable variant of the question "How can a space be tiled by replicas of a set?" has been discussed in some form or another. The sort of spaces considered have included elliptic, Euclidean and hyperbolic planes, spaces of higher dimensions, manifolds such as tori, and even algebraic structures such as groups. The sets (tiles) considered have ranged from convex polyhedra (the best behaved of all) to fractals and related sets (Mandelbrot [1977], Giles [1979]) or even to non-measurable sets with bizarre properties such as those which occur in the Hausdorff paradox. Even the word "tiling" has been used in at least two different interpretations: it means either a partition of the space into pairwise disjoint sets, or, for topological spaces, a covering of the space such that the interiors of the tiles are pairwise disjoint.

It is clearly impossible to consider all these variants here; we shall restrict ourselves to monohedral tilings of Euclidean $d$-space $E^{d}$ by tiles which are closed topological cells. The word "monohedral" means that each tile is congruent (that is, isometric) to a fixed set $S$, called the prototile of the tiling, and a "closed topological cell" is any homeomorphic image of the unit $d$-dimensional ball $\left\{\left(x_{1}, \ldots, x_{d}\right) \mid \Sigma x_{i}^{2}<1\right\}$. The word "tiling" is used in the second of the two senses mentioned above. Our motivation in restricting attention to this case is that it is clearly what Hilbert had in mind when he formulated his eighteenth problem. If $S$ is the prototile of a monohedral tiling $\mathcal{T}$ then we say that $S$ admits the tiling $\mathcal{T}$.

Even with the restrictions just stated, there are many different kinds of tilings; we shall now discuss these, and introduce the terminology needed to make our statements precise. Some of the terms used are now in reasonably widespread use-others are introduced here for the first time, in the hope that precise and convenient terminology will contribute to clear thinking.

To begin with we discuss variants that arise if we place restrictions on the mutual positions of the tiles. A tiling $\mathcal{T}$ is isohedral if the symmetry group of $\mathcal{J}$ is transitive on the tiles-in other words, the tiles belong to one transitivity class. In this case we shall also say that the prototile is isohedral. Clearly every isohedral tiling is monohedral. If a prototile admits a monohedral tiling but no isohedral tiling, then it is called anisohedral. An isohedral convex polyhedron is called a stereohedron. If a stereohedron admits a tiling in which the translational symmetries act transitively on the tiles, it is called a parallelohedron. As we shall see later, a powerful method of constructing stereohedra is by taking the Dirichlet regions of a dot pattern,-that is, of a discrete system of points in $E^{d}$ on which the symmetries of the system act transitively. In crystallographic literature such stereohedra are known as "Dirichlet domains" or "Wirkungsbereiche"-we shall call them plesiohedra.

Some tilings by convex polyhedra have the property of being face-to-face (edge-to-edge in the 2-dimensional case). This simply means that the intersec- 


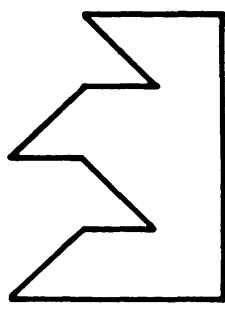

(a)

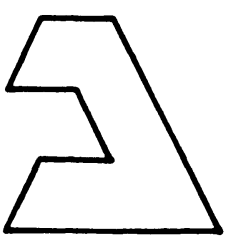

(e)

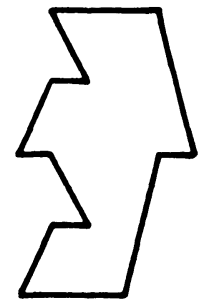

(b)

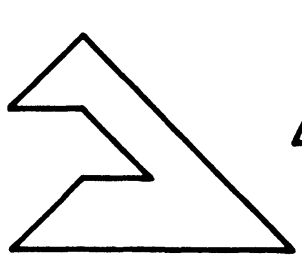

(c)

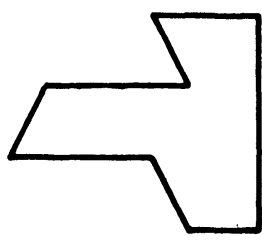

(d)

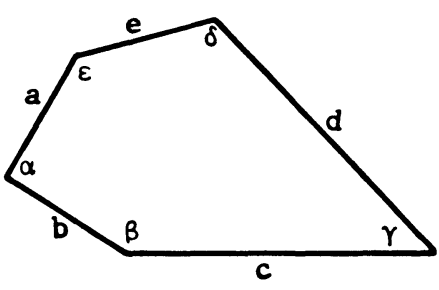

$$
\begin{array}{ll}
a=b=e & c=d \\
\alpha+\beta+\delta=2 \pi & a=2 \gamma
\end{array}
$$

(f)

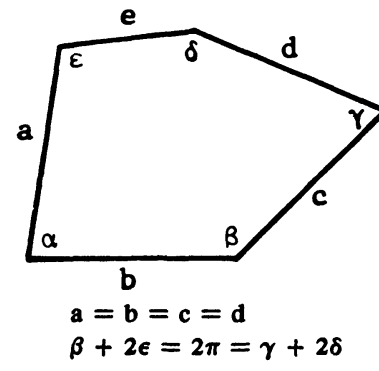

(g)

FIGURE 1. Examples of anisohedral tiles. The one shown in (a) is the first such tile discovered (Heesch [1935]); the tiles in (b), (c), (d) are from Heesch, Heesch and Loef [1944], Heesch [1968a] and Milnor [1976], respectively; the tile in (e) was communicated to us by Ludwig Danzer, who attributed it to Martin Kneser. A more detailed discussion of such tiles is given in Heesch [1976b, Chapter 4]. The tile in (f) was found by Kershner [1968], that in (g) by Marjorie Rice (see Schattschneider [1978]); these two are prototiles of monohedral edge-to-edge tilings, and the first uses only directly congruent tiles. The reader may amuse himself establising that each of these prototiles is anisohedral; the discovery of tilings will probably be experienced as an easier task than proving that no isohedral tiling is possible.

tion of any two tiles is either empty or is a face (of any dimension) of each. The three regular tilings of the plane are familiar examples of edge-to-edge tilings. (Some authors include in the definition of stereohedra the requirement that the tiling is face-to-face; we do not make this assumption.)

The second part of Hilbert's eighteenth problem can now be formulated as follows:

(A) Does there exist an anisohedral polyhedron in Euclidean 3-space?

From the phrasing of the problem as well as from other evidence (see Grünbaum and Shephard [1978]) it seems likely that Hilbert believed that there exist no anisohedral tiles in the plane. If so, he was mistaken. After Reinhardt [1928] found the affirmative solution to Problem (A), Heesch [1935] discovered the anisohedral planar prototile shown in Figure 1(a). In Figures 1(b), (c), (d), (e) we show anisohedral prototiles of a similar nature. But "stronger" examples exist; Kershner [1978] discovered three kinds of anisohedral convex pentagons which admit edge-to-edge tilings of the plane (one is shown in Figure 1(f)). A different kind of such pentagons was found by $M$. Rice (see Figure $1(\mathrm{~g})$ ). Of course, by taking prisms based on anisohedral polygons it is easy to see that the $d$-dimensional version of Problem (A) has an affirmative solution for all $d>2$. The three recent surveys of developments related to Hilbert's problems (Aleksandrov [1969], Browder [1976], Kaplansky [1977]) are singularly unhelpful in their discussion of 
Problem (A). In the first, Delone [1969] stops with the mention of Reinhardt [1928]. In the second, Milnor [1976] mentions Heesch's work but not Kershner's, and existence of anisohedral convex polygons is stated to be an open problem. In the third, in which the appropriate chapter is entitled "Building up of space from congruent polyhedra", the discussion deals exclusively with crystallographic groups, and not a single sentence reports results on polyhedra or tilings!

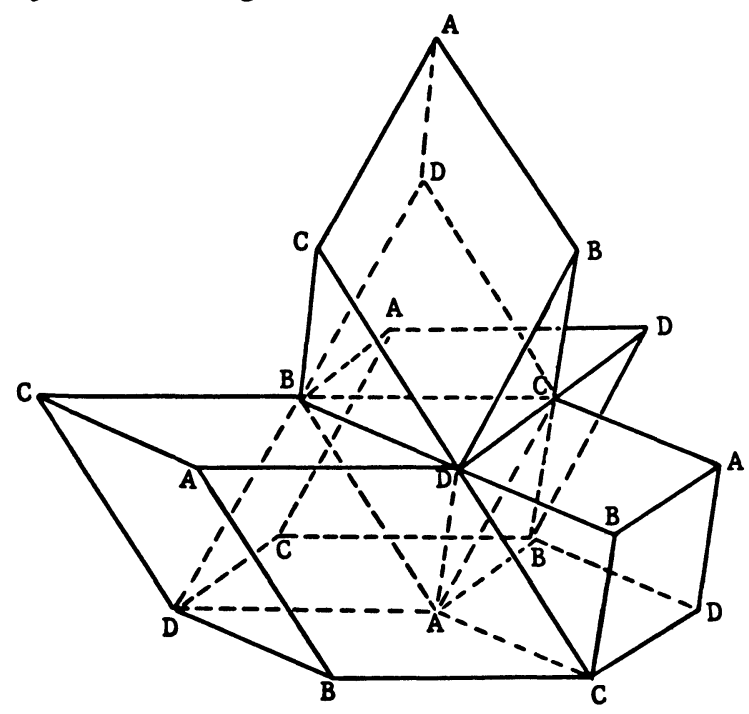

Figure 2. A monohedral tiling of $E^{3}$ by parallelepipeds. Each face of the prototile is a rhomb with angles $60^{\circ}, 120^{\circ}, 60^{\circ}, 120^{\circ}$, and the tiles occur in four aspects. First four tiles (one of each aspect) are assembled as shown in the diagram; then this "shape" will tile $E^{3}$ using translations only. As an indication how to do this we have labelled each vertex $A, B, C$ or $D$; vertices which meet must bear the same label. This tiling occurs in two enantiomorphous forms and has the remarkable property that the intersection of any two adjacent tiles is not a two-dimensional face of either. The tiling was discovered independently by Ludwig Danzer and the authors in 1975.

Tilings by congruent cubes. A classical result of Minkowski [1907, p. 74] establishes that (for $d=2$ and $d=3$ ) in every monohedral tiling of $E^{d}$ by congruent $d$-dimensional cubes in which a group of translations acts transitively, there exists a "stack" of cubes in which each two adjacent cubes intersect in a whole $(d-1)$-dimensional face; Minkowski conjectured that this statement remains valid for all $d$. Proofs of the Minkowski conjecture for $d=4,5,6$ were given by Jansen [1909], for $d<9$ by Perron [1940b], [1941], and in all generality by Hajós [1942]. The following generalization of the problem was proposed by Keller [1930]:

(B) Does there exist, in every monohedral tiling of $E^{d}$ by congruent d-dimensional cubes, a tile which shares a complete $(d-1)$-dimensional face with another tile?

Perron [1940a] established that the answer to Problem (B) is affirmative for $d<6$; there seem to have been very few new results concerning Problem (B) in the intervening forty years. For a discussion of these problems and results see Stein [1974], Seitz [1975], as well as the papers of Robinson [1979], [1980] in which the analogues of the Minkowski conjecture and of Problem (B) for 
tilings of multiplicity exceeding 1 are considered. For a graph-theoretic reformulation of Problem (B) see Lawrence [1980].

If in Problem (B) "congruent $d$-cubes" are replaced by "congruent $d$-parallelepipeds" (that is, affine images of a $d$-cube) the situation changes thoroughly. It is not hard to verify that the answer is still affirmative for $d=2$, but for $d=3$ it is negative! This is shown by the tiling in Figure 2, in which the prototile has as faces six congruent rhombs. It illustrates also the fact that even in such a relatively simple case, tilings of $E^{3}$ are difficult to visualize, and even harder to depict diagrammatically.

The great variety of tilings using parallelepipeds is illustrated by the observation of Heesch [1934] (see also Zamorzaev [1965]) to the effect that in $E^{3}$ there exist infinitely many topologically inequivalent isohedral tilings of this kind (see Figure 3).

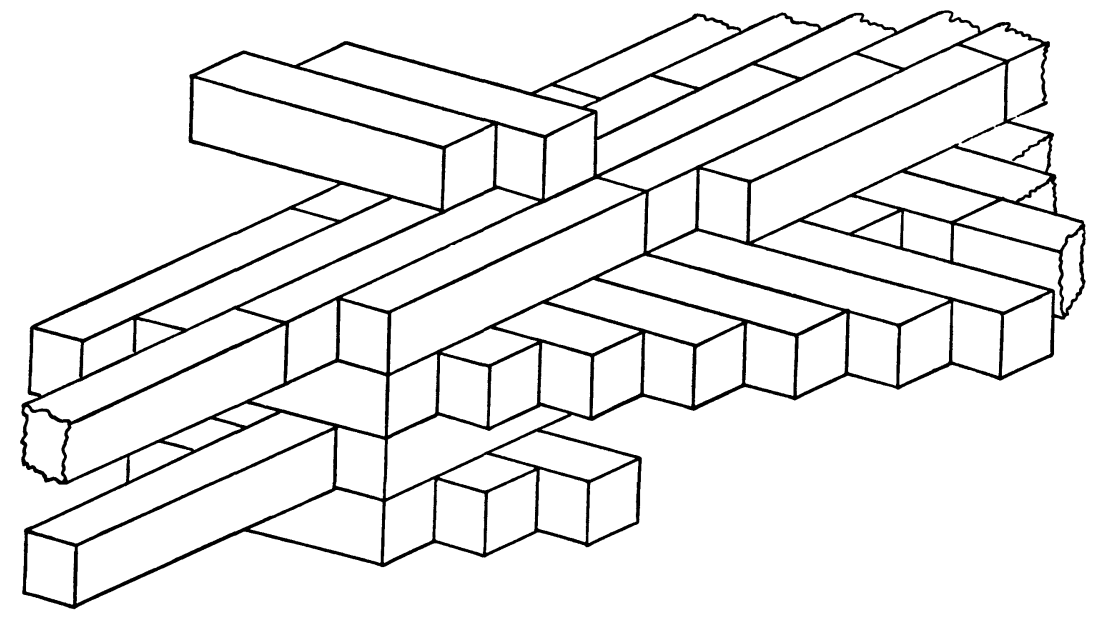

FIGURE 3. A method of constructing an infinite number of topologically inequivalent isohedral tilings with prototile combinatorially equivalent to the cube; it is due to Heesch [1934]. Staggered horizontal stacks of boxes of size $k \times 1 \times 1$ form layers, which are placed on top of each other with the long sides alternating in two mutually perpendicular directions. Since each tile in such a tiling is adjacent to $2 k+6$ other tiles along two-dimensional common boundaries, different values of $\boldsymbol{k}$ correspond to distinct topological types of tilings.

Aperiodic tilings. The following problem is undecided even for $d=2$.

(C) Does there exist a prototile $P$ which admits monohedral tilings of $E^{d}$, but no such tiling possesses a translation as a symmetry?

It seems likely that the answer to this question for $d=2$ is in the negative, though the corresponding problem for dihedral tilings (that is, tilings in which the tiles are of two different kinds) has an affirmative answer. These results are related to the well-known "Penrose tiles" (see Gardner [1977], Penrose [1978]; a detailed account of "aperiodic tilings" appears in Grünbaum and Shephard [1980a, Chapter 10]). Problem (C) is unsolved even in the very special case in which $d=2$, the prototile $P$ is a convex polygon, and the tiling is required to be edge-to-edge and to use only directly congruent copies of $\boldsymbol{P}$.

The fundamental problem underlying all these questions is, of course, to determine all prototiles of monohedral tilings in $E^{d}$. Stated in this generality 
it is obvious that there is no reasonable expectation of a solution. For this reason we shall mostly restrict our discussion in the following pages to the case in which the prototile is convex:

(D) Determine all convex polyhedra which are prototiles of monohedral tilings of $E^{d}$.

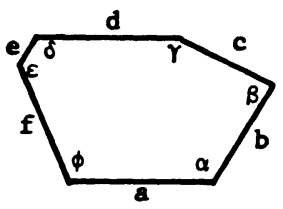

$a=d \quad \alpha+\beta+\gamma=2 \pi$

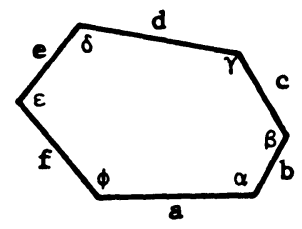

$a=d \quad c=e$ $\alpha+\beta+\delta=2 \pi$

(a)

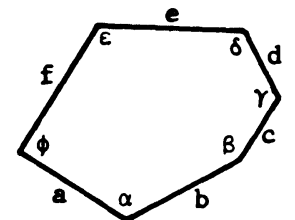

$\mathrm{a}=\mathrm{b} \quad \mathrm{c}=\mathrm{d} \quad \mathrm{e}=\mathrm{f}$ $\alpha=\gamma=\epsilon=2 \pi / 3$

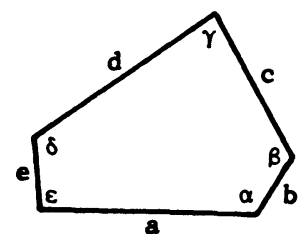

$a=d \quad \alpha+\beta+\delta=2 \pi$

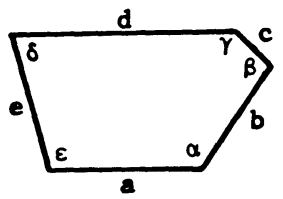

$\alpha+\beta+\gamma=2 \pi$

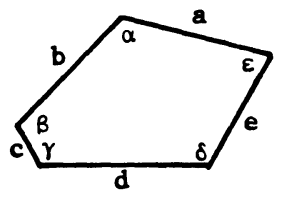

$\mathrm{a}=\mathrm{b} \quad \mathrm{d}=\mathrm{c}+\mathrm{e}$

$\alpha=\gamma=\delta=2 \pi / 3$
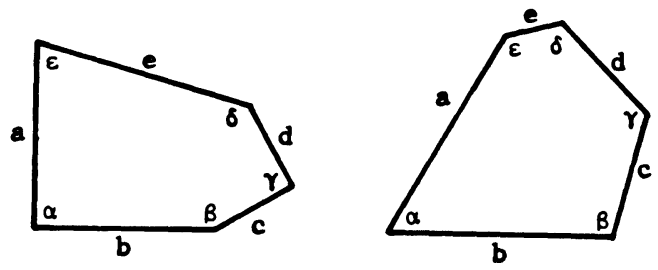

$$
\begin{gathered}
a=b \quad c=d \\
\alpha=\gamma=\pi / 2
\end{gathered}
$$$$
\mathrm{a}=\mathrm{b} \quad \mathrm{c}=\mathrm{d}
$$$$
\alpha=\pi / 3 \quad \gamma=2 \pi / 3
$$

(b)

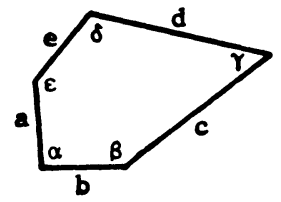

$\mathrm{a}=\mathrm{b}=\mathrm{e} \quad \mathrm{c}=\mathrm{d}$

$\alpha=2 \gamma \quad \gamma+\epsilon=\pi$

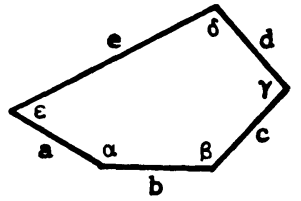

$\mathrm{a}=\mathrm{b}=\mathrm{c}=\mathrm{d}$

$a=b=c=d$

$\alpha+2 \delta=2 \pi \quad 2 \beta+\gamma=2 \pi \quad 2 \alpha+\beta=2 \pi \quad \gamma+2 \delta=2 \pi$

(c)

FIGURE 4 (first part). 


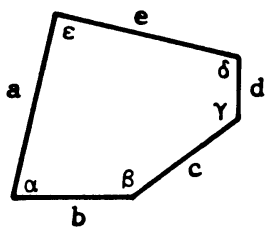

$$
\begin{aligned}
& a=e=b+d \quad 2 \beta-\delta=\pi \quad 2 \gamma+\delta=2 \pi \quad \epsilon=\pi / 2 \\
& \alpha+\delta=\pi
\end{aligned}
$$

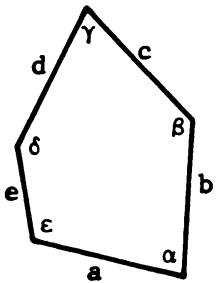

$a=b=c=d$

$\beta+2 \epsilon=2 \pi$

$\gamma+2 \delta=2 \pi$

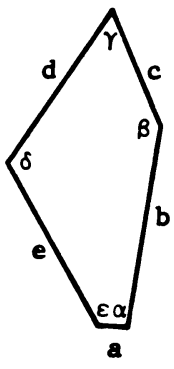

$2 \mathrm{a}+\mathrm{c}=\mathrm{d}=\mathrm{e}$

$\alpha=\pi / 2 \quad \gamma+\epsilon=\pi$

$2 \beta+\gamma=2 \pi$

(d)

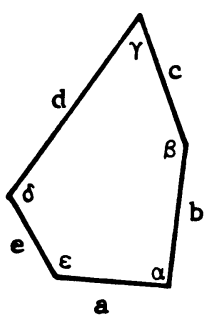

$2 \mathrm{a}=\mathrm{c}+\mathrm{e}=\mathrm{d}$

$\alpha=\pi / 2 \quad \gamma+\epsilon=\pi$

$2 \beta+\gamma=2 \pi$

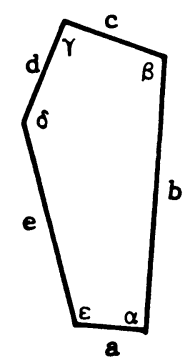

$c=\mathrm{d} \quad 2 \mathrm{c}=\mathrm{e}$

$\beta=\epsilon=\pi-\delta / 2$

$\alpha=\gamma=\pi / 2$

(e)

Figure 4 (second part).

Figure 4. The known types of convex prototiles of monohedral planar tilings. All triangles and all quadrangles are stereohedra, and all convex hexagonal tiles are of one of the three types indicated in (a); these, and the five types of pentagons in (b), have been known since Reinhardt [1918]. (It should be noted that the types here, as well as the ones below, are not mutually exclusive.) All the known convex pentagonal prototiles are shown in (b), (c), (d) and (e), but the question whether or not other such tiles exist is still undecided. The three types in (c) are due to Kershner [1968], that in (d) to Richard James, and the four in (e) to Marjorie Rice (see Schattschneider [19781). Near each tile the conditions characterizing the type have been indicated; Greek letters indicate angles, lower case characters lengths of sides. For vigorous mental exercise the reader is urged to verify that each of the polygons shown admits a monohedral tiling.

Tilings of the plane. Reinhardt [1918] thought that he had completely solved Problem (D) for $d=2$, although he stopped short of flatly asserting this as a fact. However, his vaguely formulated reservations were mostly overlooked, and for a long time it was "known" that all types of convex prototiles of monohedral tilings of the plane had been determined by Reinhardt. But Kershner [1968] showed that Reinhardt's list of types was incomplete, and he produced an enlarged list for which he claimed completeness; three of his types are examples of anisohedral planar tiles. (Many other publications deal with special cases of Problem (D) in the plane or with related matters; references to them can be found in Schattschneider [1978] or in Grünbaum and Shephard [1978], [1980a, Chapter 9].) Following an exposition of Kershner's enumeration of types by Gardner [1975a], the incompleteness of Kershner's solution was pointed out by a reader (see Gardner [1975b]). Detailed accounts of the "new" types of planar prototiles are given 
in Schattschneider [1978] and in Grünbaum and Shephard [1980a]; they are illustrated in Figure 4. After two false claims, nobody seems to be claiming now that Problem (D) has been completely solved in any number of dimensions exceeding 1; it would be nice to see this question settled, at least for $d=2$.

The determination of all planar stereohedra is much easier; it is contained in a classification of isohedral tilings by convex polygons given in Grünbaum and Shephard [1978] (see also Grünbaum and Shephard [1980a, Chapter 9], where related questions are considered as well).
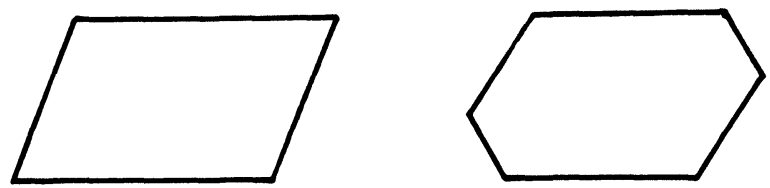

(a)
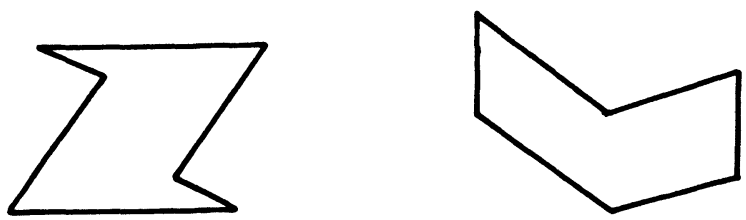

(b)

FiouRE 5. Planar parallelohedra ("parallelogons") are either quadrangles or hexagons; in each case a necessary and sufficient condition for the polygon to be a parallelogon is that opposite sides be parallel and equal in length. Hence the two tiles in (a) are representative of the most general parallelogons. If convexity is not required, there exist many other types of prototiles that admit monohedal tilings on which translational symmetries act transitively; two examples are shown in (b), a method for constructing all such tiles is illustrated by an example in Figure 6.

The determination of all planar parallelohedra (which are better known as "parallelogons") is even simpler; it was carried out by Fedorov [1885] (see Figure 5). Fedorov [1899] mentions also non-convex polygons as parallelogons; some other authors (see, for example, Šubnikov and Kopcik [1972]) do not mention convexity in dealing with parallelogons, but seem to be tacitly assuming it. A complete list of prototiles (polygonal or not), that admit monohedral tilings in which translational symmetries act transitively on the tiles, can be obtained from the classification of isohedral tilings of the plane given in Grünbaum and Shephard [1977]; it consists of the prototiles of the tilings described there as being of types IH 1, 8, 10, 11, 12, 14, 17, 18, 20,41, $57,62,64,68,72,74$, and 76 (see Figure 6). An analogous determination of all planar plesiohedra can be found in Delone, Dolbilin and Štogrin [1978].

Monohedral tilings of $E^{d}$ by convex polyhedra. Since Problem (D) has not been solved in the plane, it seems quite hopeless to ask for a solution in $d>3$ dimensions. Consequently, more modest problems-such as the followinghave attracted considerable attention.

(E) Determine all simplexes (or, indeed, $n$-faced polyhedra for small values of $n$ ) which are prototiles of monohedral tilings of $E^{d}$. 
(F) Determine all combinatorial types of convex polyhedra which are prototiles of monohedral tilings of $E^{d}$.

(G) Determine the least upper bound for the number of $(d-1)$-dimensional faces of convex polyhedra which are prototiles of monohedral tilings of $E^{d}$.

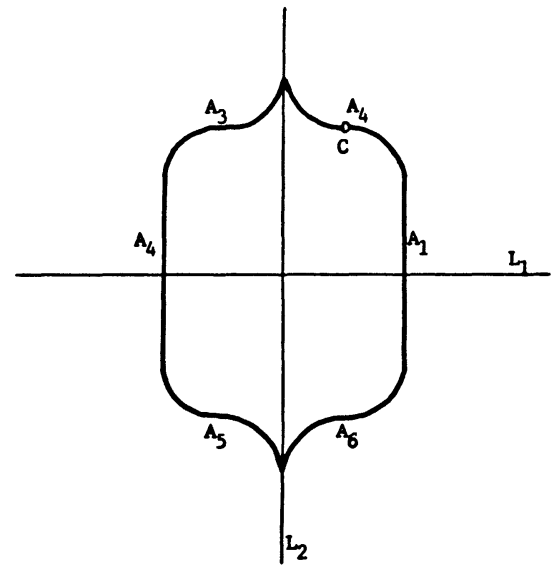

Figure 6. An example of the construction of prototiles of monohedral tilings that admit a tile-transitive group of translational symmetries ("generalized parallelogons"). Shown here is the case of prototiles $P$ that admit tilings of type $\mathrm{IH} 17$ in the notation of Grünbaum and Shephard [1977]. Each such $P$ admits two mutually perpendicular lines of reflective symmetry $\left(L_{1}\right.$ and $\left.L_{2}\right)$. The boundary of $P$ is a simple curve which consists of six consecutive arcs $A_{1}, A_{2}, A_{3}, A_{4}, A_{5}, A_{6}$ such that: (i) $A_{1}$ and $A_{4}$ are equal straight-line segments each with perpendicular bisector $L_{1}$; (ii) $A_{2}$ is a simple arc which has a center of symmetry $C$, does not meet $L_{1}$, and has one endpoint at an endpoint of $A_{1}$, the other on $L_{2}$; (iii) $A_{3}$ is the reflection of $A_{2}$ in $L_{2}, A_{6}$ its reflection in $L_{1}$, and $A_{5}$ its reflection in $L_{1}$ and $L_{2}$. Each of the other types of isohedral tilings listed in the text leads analogously to a family of prototiles with the desired properties.

None of these problems has been completely solved for any value of $d>3$. In connection with Problem (E), Goldberg [1972], [1974a], [1974b], [1976], [1977], [1978], [1979] has obtained many examples of tetrahedra, pentahedra and other polyhedra which admit tilings of $E^{3}$, but there is no proof or claim that his lists are complete. From Goldberg's compilations it follows that each of the two combinatorial types of pentahedra in $E^{3}$ has representatives which are stereohedra. Similarly, for six of the seven combinatorial types of hexahedra in $E^{3}$ it is not hard to find representatives which are stereohedra. However, it appears to be unknown whether there exists any stereohedron which is a pentagonal pyramid. It is not even known whether there is any (locally finite) tiling of $E^{3}$ in which each tile is a pentagonal pyramid, without any requirement of congruence! Examples of monohedral tilings of higher-dimensional spaces by simplices were given by Baumgartner [1968], [1971], Danzer [1968] and Debrunner [1980].

Parallelohedra. As a partial solution of Problem (F), Fedorov [1885] determined the five possible combinatorial types of three-dimensional parallelohedra (see Figure 7). All five are vector sums of line segments (zonohedra), but the analogous assertion is not true for parallelohedra in $d>4$ dimensions (Coxeter [1962]). In a later paper, Fedorov [1899] showed that according to a more refined classification scheme there are 37 distinct 
types of 3-dimensional parallelohedra (compare Delone, Galiulin and Štogrin [1979]). Among Fedorov's parallelohedra there are 24 types which are plesiohedra (see Delone [1932], Delone, Padurov and Aleksandrov [1934, p. 179]).
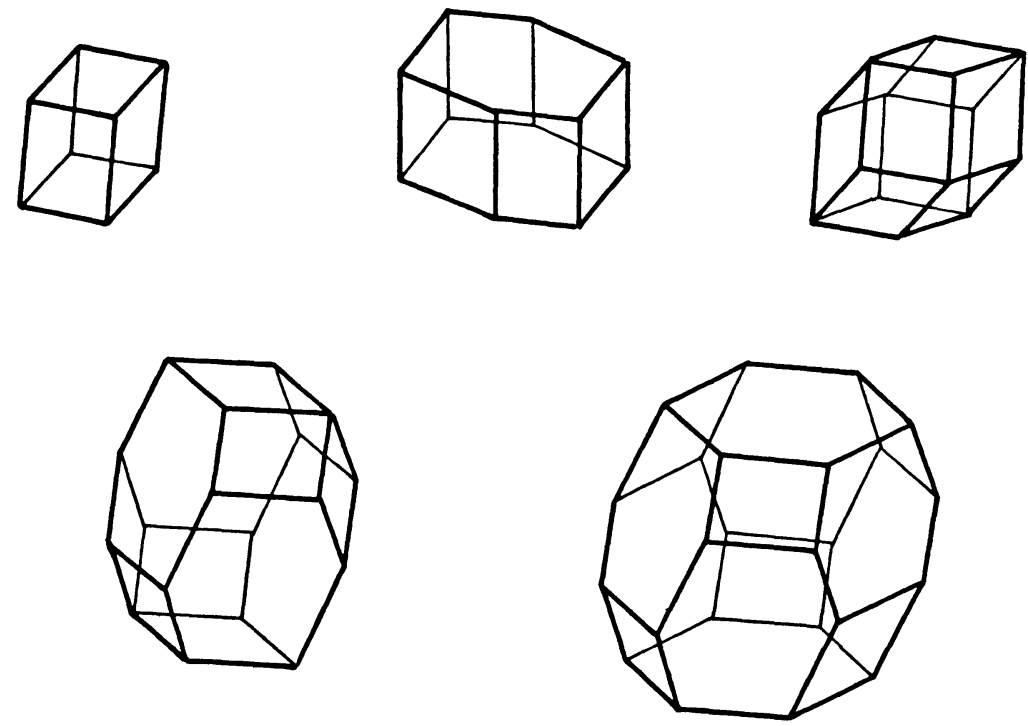

FIOURE 7. The five combinatorial types of parallelohedra in $E^{3}$.

The largest number of faces among three-dimensional parallelohedra is 14 , and for a long time this was thought to be the maximal possible number of faces in any stereohedron (see, for example, Baily [1908] or its review Lampe [1911], or Smith [1978]). Minkowski [1897] established that parallelohedra in $E^{d}$ have at most $2\left(2^{d}-1\right)$ faces of dimension $d-1$.

Stereohedra. Concerning Problem (G), the only general result available is due to Delone [1961] (see also Štogrin [1973]). To formulate this we recall that two congruent sets are said to have the same aspect if one is a translate of the other. Delone's theorem is as follows:

The number of $(d-1)$-dimensional faces of a stereohedron $P$ in $E^{d}$ is at most $2^{d}(1+a)-2$, where $a$ is the number of aspects of $P$ in an isohedral tiling of $E^{d}$.

In the case $d=3$ the maximal number of aspects is 48 , since this is the maximal number of inequivalent points in a translational fundamental domain of any dot pattern in $E^{3}$ (see Henry and Lonsdale [1965]). Consequently a stereohedron in $E^{3}$ has at most 390 two-dimensional faces. This estimate is almost certainly much too high; we now turn to survey in some detail the presently available results. Since we are concerned only with the case $d=3$, we shall simplify the wording by using "face" to mean 2-dimensional face" and "space-filler" to mean "convex polyhedron which admits a monohedral tiling of $E^{3 \prime}$.

We have already mentioned a 14-faced space-filler. A 16-faced space-filler was described by Föppl [1916]; it can be obtained from the "uniform tiling" 


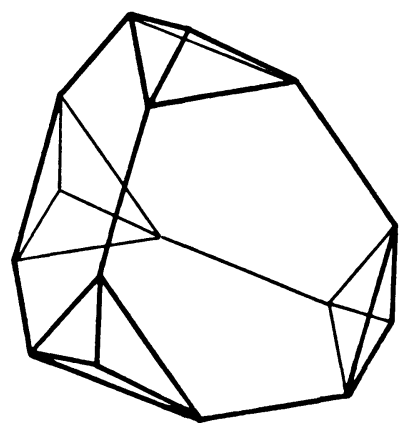

FIGURE 8. The 16-faced space-filler discovered by Föppl [1914].

of $E^{3}$ by regular tetrahedra and truncated tetrahedra by partitioning the tetrahedra equally among the neighboring truncated tetrahedra (see Figure 8). Even more simply it can be described as the plesiohedron obtained from the dot pattern usually called the "diamond lattice". It has been rediscovered several times and in many accounts it is presented as being the space-filler with the maximal possible number of faces (see, for example, Critchlow [1970], Williams [1972], Coxeter [1978], Goldberg [1979], Baracs [1979]).

Plesiohedra with 17 and with 18 faces were described by Nowacki [1935]. A more detailed investigation of the same kinds of plesiohedra by Löckenhoff and Hellner [1971] corrected a number of errors and led to two different combinatorial types with 17 faces and one with 18 faces; these correspond to certain special dot patterns ("invariant cubic lattice complexes" in the crystallographic terminology). The 18-faced plesiohedron is illustrated in Figure 9(a).

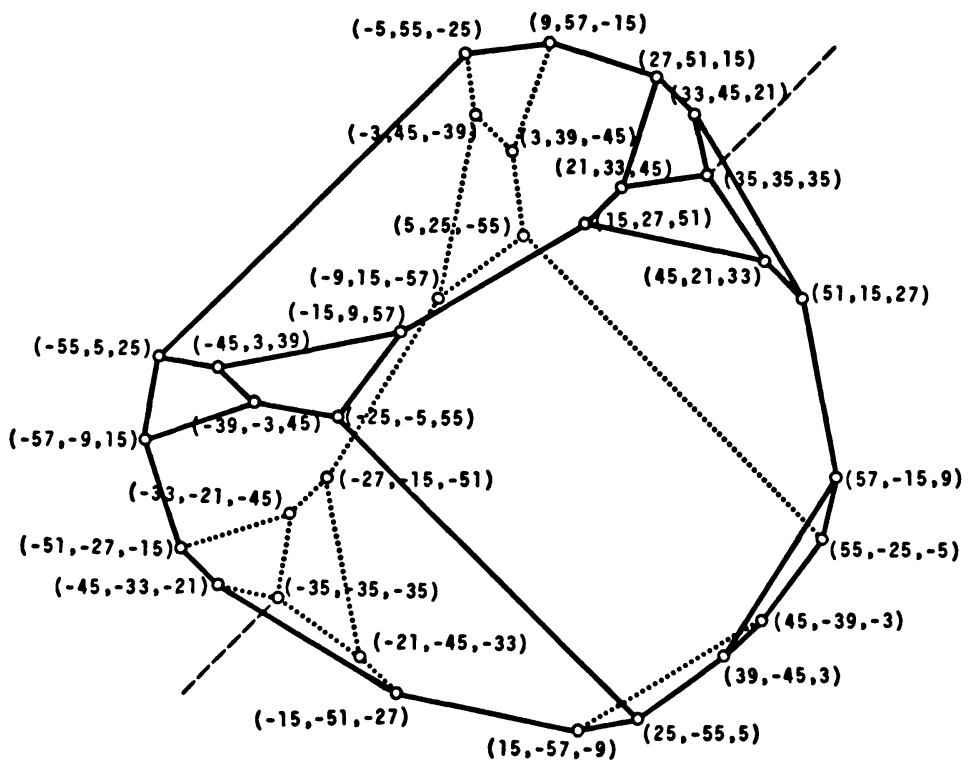

(a)

FIGURE 9 (first part) 


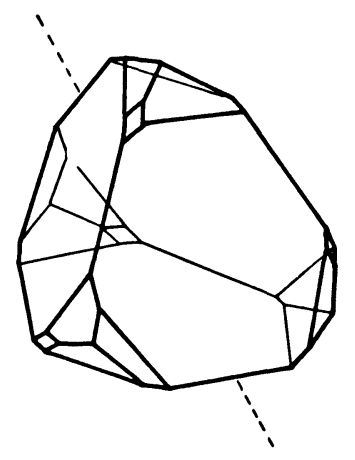

(b)
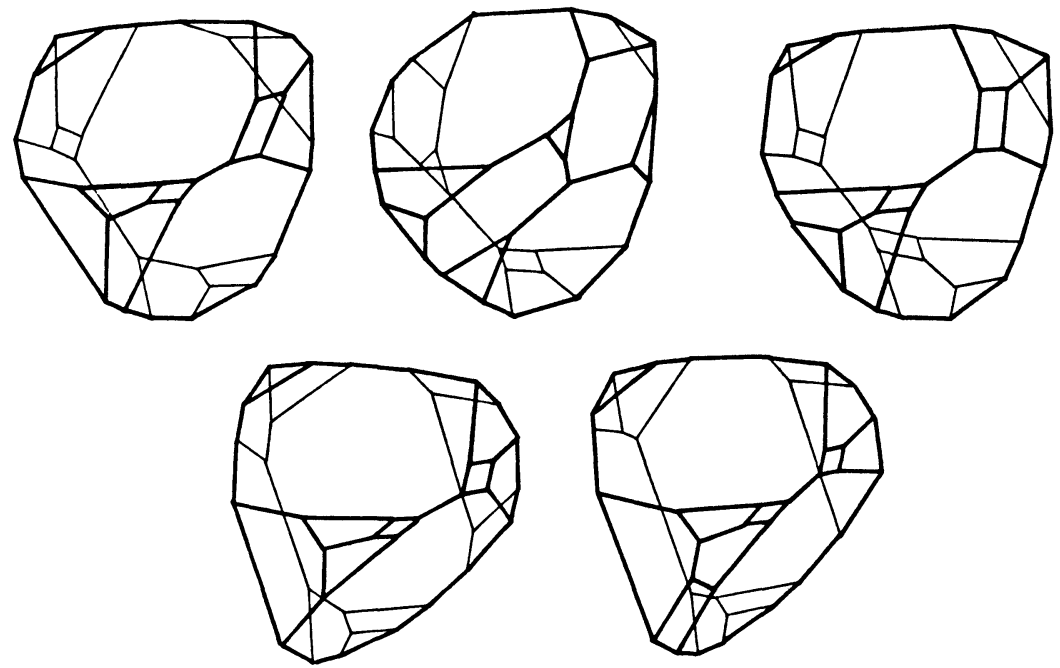

(c)

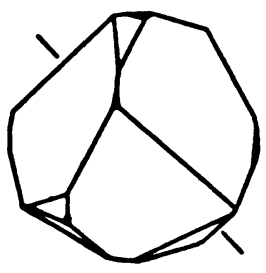

22

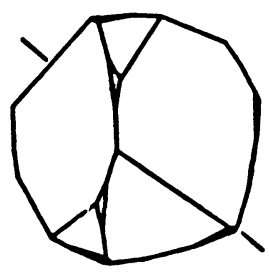

22

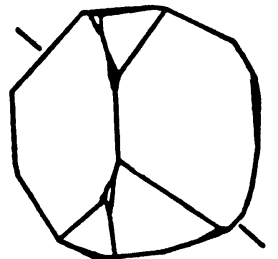

24

(d)

FIGURE 9 (second part)

Figure 9. (a) A plesiohedron with 18 faces from Löckenhoff and Hellner [1971]; (b) a 20-faced plesiohedron described by Smith [1965]; (c) five 20-faced plesiohedra found by Stogrin [1968], [1973]; (d) one 24-faced and two 22-faced plesiohedra, from Koch and Fischer [1972]. 
A 20-faced plesiohedron was discovered by Smith [1965] (see Figure 9(b)); it corresponds to a "perturbed diamond lattice" and tiles using two aspects of the prototile.

Stogrin [1968], [1973] found five (infinite families of metrically different) plesiohedra with 20 faces (see Figure 9(c)), as well as many other (families of, and individual) plesiohedra with 19,18,17 or fewer faces; they all tile with $a=2$ aspects.

It is interesting to observe that Delone's formula for $d=3$ and $a=2$ yields an upper bound of 22 faces; it is still undecided whether this bound is attained.

Extending the investigations of Löckenhoff and Hellner from "invariant cubic complexes" to "univariant" and "bivariant" ones, Koch [1972] found two families of plesiohedra with 23 faces (together with three families having 22 , two having 21 , and many families of types with a smaller number of faces). Similar investigations of those "tetragonally distorted cubic diamond" dot patterns (with symmetry group $P 4_{1}$ in the notation of the "International Tables" (Henry and Lonsdale [1965])) which correspond to sphere packings, by Koch and Fischer [1972], led to a 2-parameter family of 24-faced plesiohedra (as well as to 33 other families of types of plesiohedra with 8 to 22 faces). All the plesiohedra found by Koch and Fischer tile using only directly congruent copies of the prototiles. Examples of some of these plesiohedira are shown in Figure 9(d).
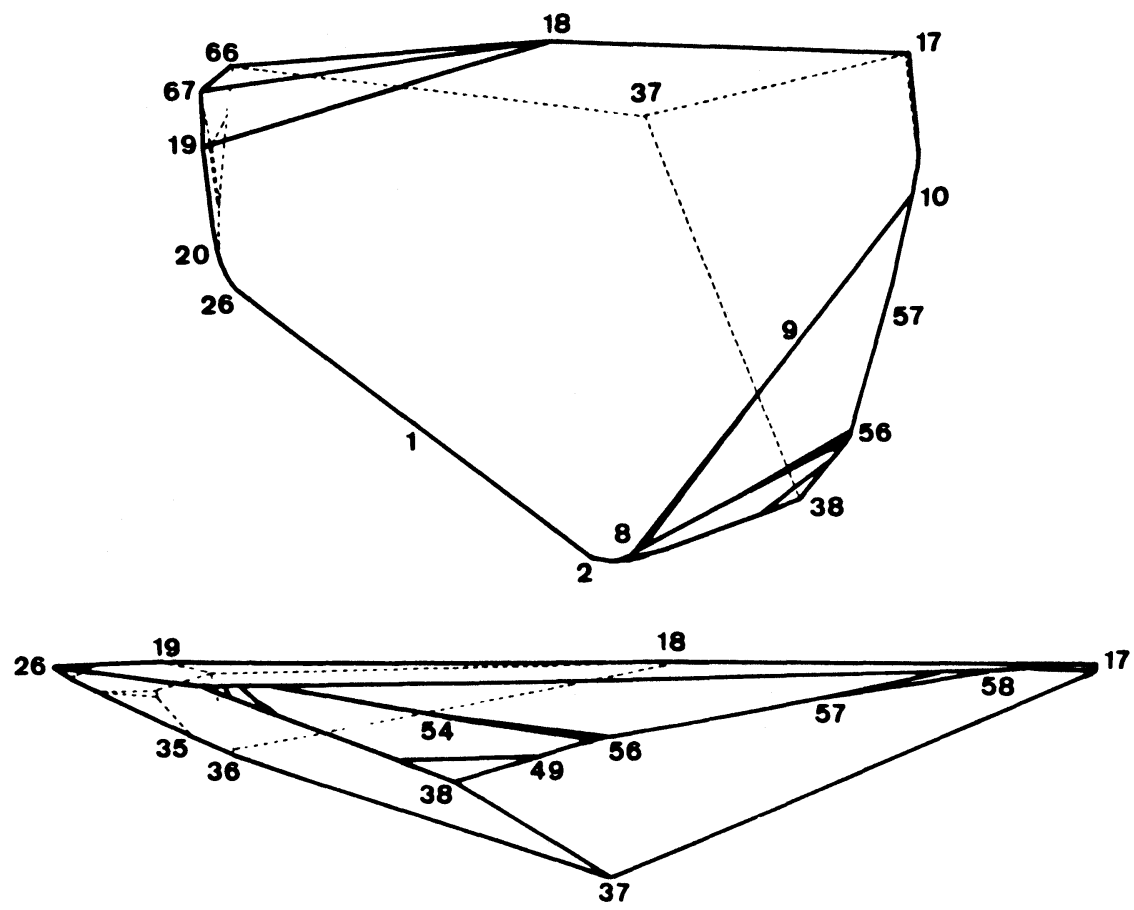

FIGURE 10. Two views of a 38-faced plesiohedron with 70 vertices, discovered by Engel [1980]; some of the vertices are numbered to simplify their recognition here and in Figure 11(a). 
Surveying the known results, Brunner and Laves [1978] conjectured that 26 $\left(=3^{3}-1\right)$ is an upper bound for the number of faces of a space-filler in $E^{3}$ (and that $3^{d}-1$ is a similar bound in $E^{d}$ ). At a crystallographic conference in August 1979 the discovery of a 26-faced space-filler was announced by Fischer [1979]. However, the conjecture-as well as the less definite feelings of many investigators-were completely upset by recent results of Engel [1980]. Engel discovered 172 combinatorial types of plesiohedra, each with realizations depending on three real-valued parameters, with 17 to 38 faces, the maximal number being represented by two types! With the kind permission

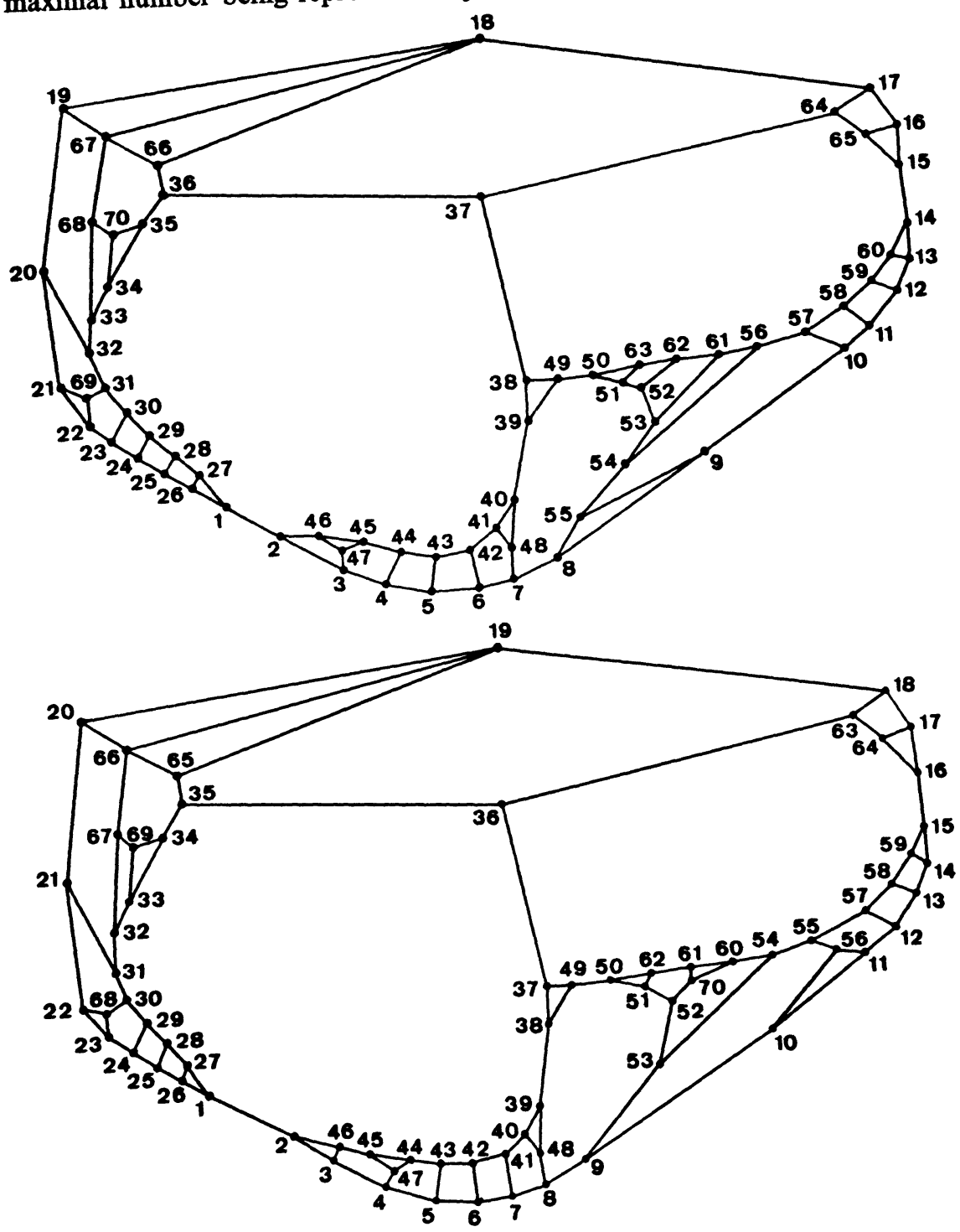

(a)

FIGURE 11 (first part) 
of Dr. Engel we reproduce in Figure 10 two sketches of one of the 38-faced plesiohedra, and in Figure 11(a) Schlegel diagrams of the two types of plesiohedra. (Dr. Engel has just informed us that he has discovered two additional types of plesiohedra with 38 faces, and two types with 37 faces; Schlegel diagrams of the 38-faced ones are shown in Figure 11(b).)

We have been considering Problems (F) and (G) in the rather restricted class of plesiohedra. The reason for this is the total absence of methods usable in more general situations. There seems to be no grounds to assume
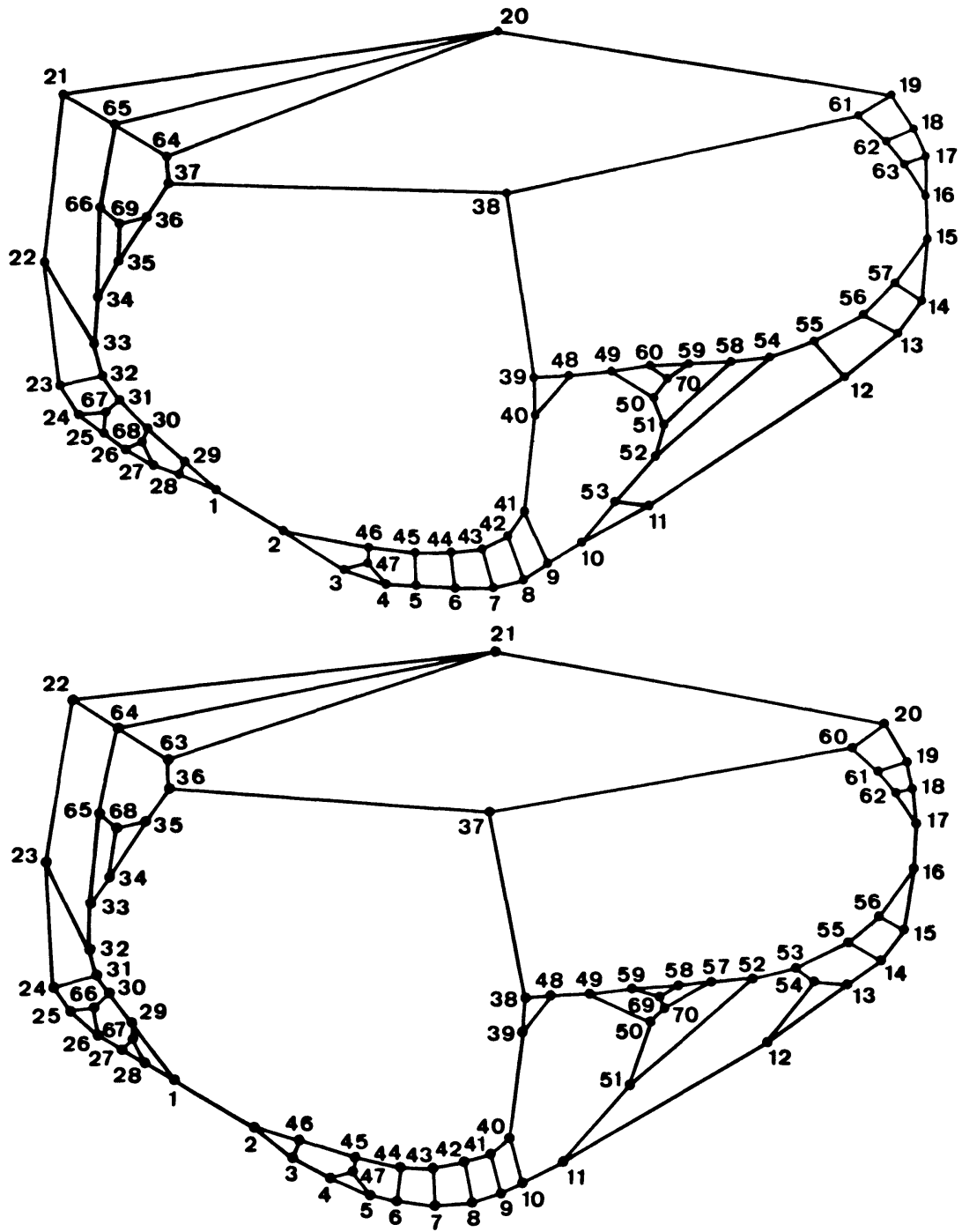

(b)

Figure 11 (second part)

Figure 11. (a) Schlegel diagrams of the two 38-faced plesiohedra of Engel [1980]; the first corresponds to the plesiohedron two views of which are shown in Figure 10. (b) Schlegel diagrams of two additional 38-faced plesiohedra found by Dr. P. Engel. 
that all stereohedra are combinatorially equivalent to plesiohedra (though no example settling this question is known), and there seems to be no practical technique available which would be guaranteed to produce-if only carried out with sufficient perseverance-all combinatorial types of stereohedra. This should be contrasted with the fact, easily provable using Tarski's decidability theorem (see Tarski [1951], Seidenberg [1954], Cohen [1969]), that all $d$ dimensional stereohedra can be "effectively" determined for each dimension d.

There is even less hope for the determination of all space-fillers. As far as we know, it is possible that Problem (G) has no finite answer, even for $d=3$.

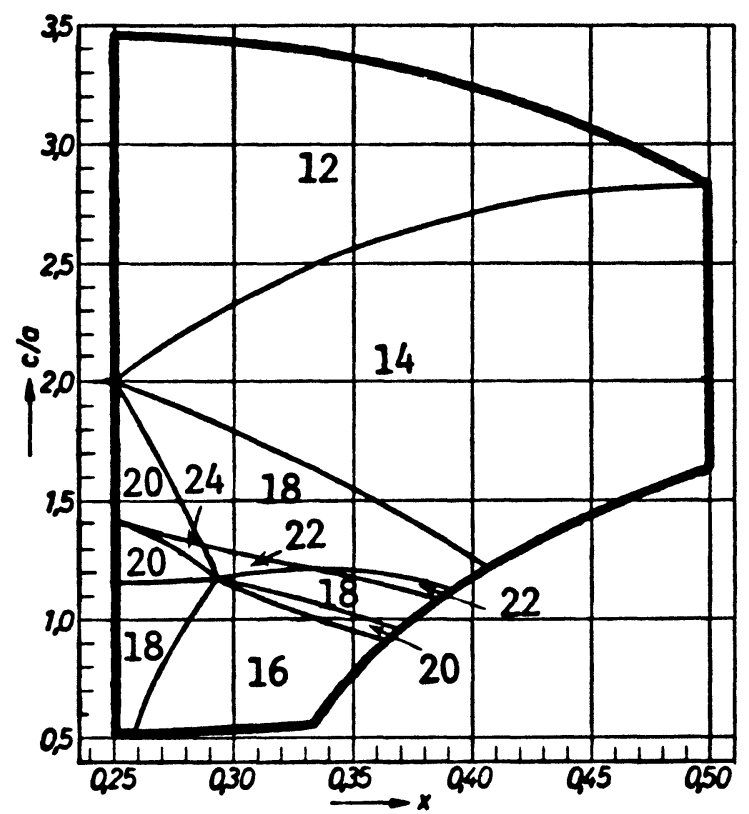

Figure 12. The "existence chart" of the types of plesiohedra considered by Koch and Fischer [1972]. The two parameters (denoted, as in the original, by $x$ and $c / a$ ) have geometric meaning for the dot pattern used to generate the tiling, but this is largely irrelevant for the present discussion. The heavy solid line surrounds the region which contains the relevant pairs $(x, c / a)$, the thin lines denote the boundaries of the regions of existence of the various combinatorial types of plesiohedra. The numbers in the regions indicate the numbers of faces of polyhedra corresponding to each region. To avoid cluttering up the diagram we have not indicated the types of polyhedra that correspond to the edges of the diagram, or to its vertices. The reader can find their full description, as well as the coordinates of the vertices and the equations of the curves separating the regions, in the original paper.

Practical computability. The methods used to obtain the plesiohedra with large numbers of faces, mentioned in the preceding section, are probably as interesting as the numbers themselves. In the investigations of Löckenhoff and Hellner, Koch, Koch and Fischer, and Engel (and in other publications) the plesiohedra are obtained from the corresponding dot patterns by the use of computers. This poses no difficulty of principal in the cases considered by Löckenhoff and Hellner [1971], since they restricted attention to dot patterns for which the points in question have well determined coordinates (forming 
an "invariant complex"). However, in the papers of Koch, Fischer and Engel the dot patterns underlying the tilings have 1, 2 or 3 variable parameters, and neither the presentation of the results, nor the verification of their completeness, is trivial.

The chief difficulty in presenting the results arises from the fact that the domain of variability of the parameters is subdivided into regions in a complicated manner, and each region corresponds to a different type of plesiohedron. As can be seen from Figures 12 and 13, a formal description of the regions by equations and inequalities would be rather cumbersome and quite useless. Hence diagrams like the "existence charts" given in these illustrations are necessary (or, at least, very helpful) if one is to understand the way in which the type of plesiohedron depends on the values of the parameters. The amount of complication in these charts convinces us that there is little likelihood of further progress in this direction without a very substantial reliance on well-programmed computers. And in those cases where there are three or more parameters, the investigation, and even the presentation of the results, appear to be extremely difficult.

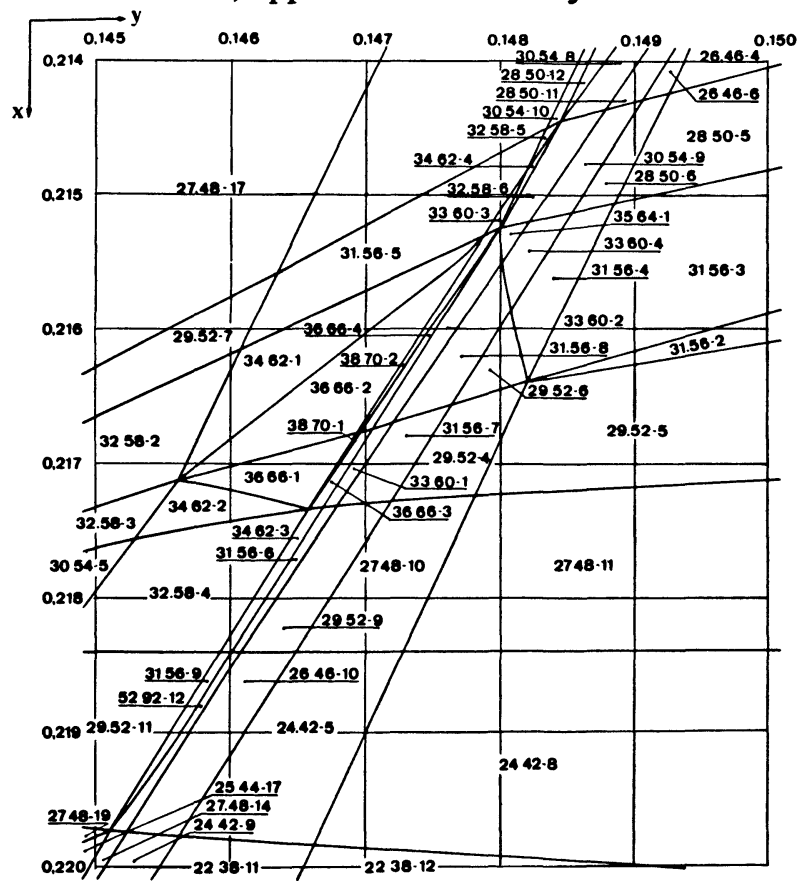

(a)

FIgURE 13 (first part)

Figure 13. The existence chart for the types of plesiohedra considered by Engel [1980]. Following preliminary investigations of the dependence of types on the parameters $(x, y, z)$ of the dot pattern, Engel restricted attention to parameter values with $z=0.0158$. In (a) the existence chart is presented for the parameter values $0.20<x<0.23$ and $0.14<y<0.20$, while in (b) the most interesting part is presented on a larger scale $(0.214<x<0.220,0.145<y<$ 0.150). In symbols $m \cdot n-p$, associated with the regions of the chart, $m$ indicates the number of faces, $n$ the number of vertices, and $p$ is a mark to distinguish different types with the same values of $m$ and $n$. 


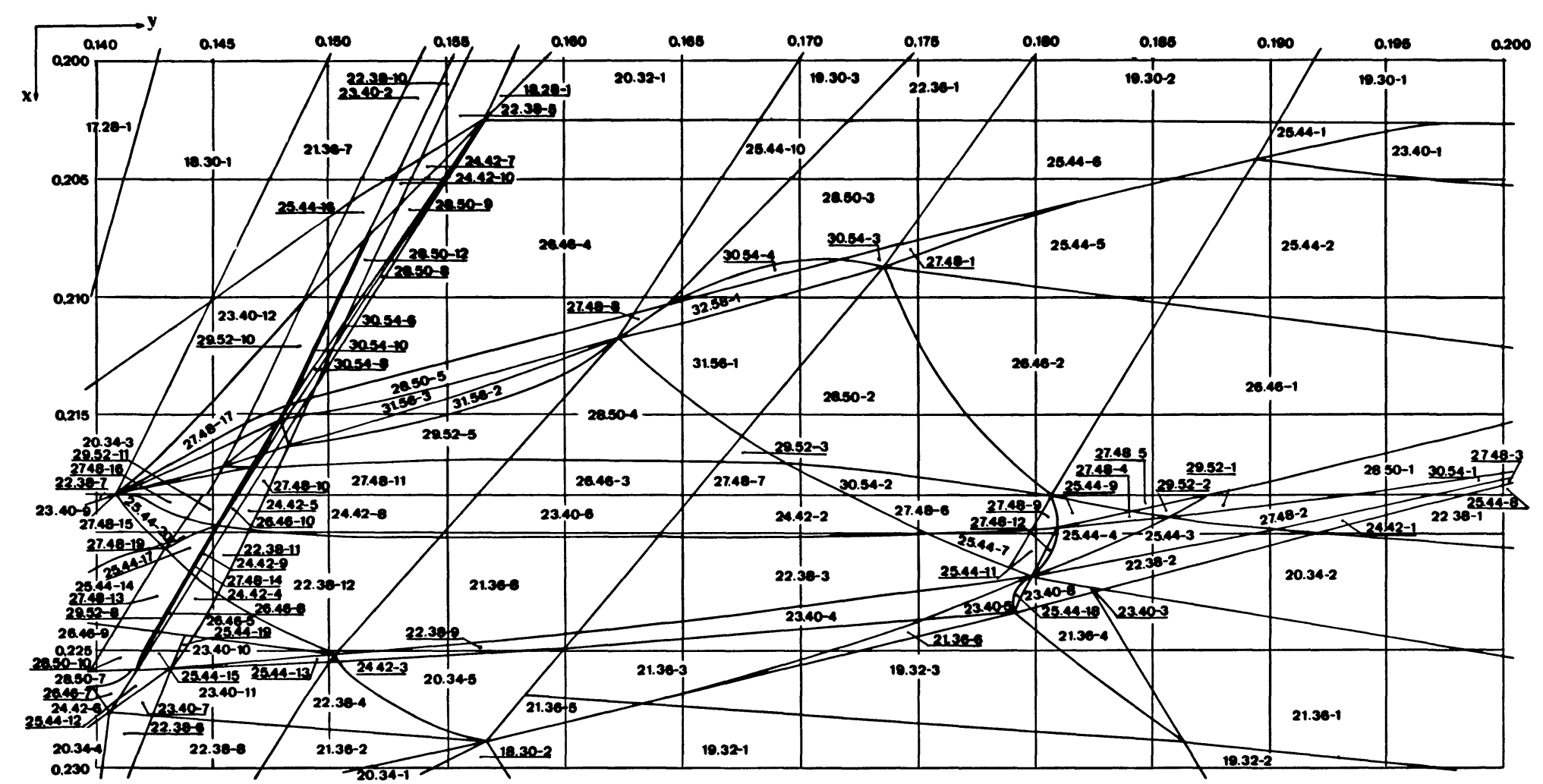

(b)

Figure 13 (second part) 
The inherent problem in these investigations stems from the fact that computers work in discrete steps, while the variability of the parameters is, of course, continuous. As described by Koch, Fischer and Engel, the determination of the types proceeds along the following general lines. First, for a relatively coarse grid of parameters of the dot pattern, the types of the plesiohedra are determined. This gives a rough idea of the various regions of the parameter space that correspond to different types of plesiohedra. This knowledge, and the underlying geometry, are then used to find an equation for the curve (or surface) that forms the boundary between adjacent regions of the parameter values. Refined grids of points near and on this curve or surface, as well as other considerations, are used to check that no subregion corresponding to a different type has been missed. Admittedly, if reasonably fine grids are used it is very likely (or even overwhelmingly likely) that all regions have been found-but there is, in principle, no possibility of attaining absolute certainty without using some additional idea or technique. At the moment, these seem to be lacking.

It should be noted that an "effective" algorithm for the determination of all types was described by Delone and Sandakova [1961]-but it appears not to be practically feasible.

In the case of stereohedra which tile with a very simple group $(P \overline{1})$, a practical method for the determination of all types was devised by Stogrin [1968], [1971]. But in other cases we are at present in the very unsatisfactory position of being "completely convinced" about the validity of certain results (such as the enumerations of types by Koch and Fischer [1972] or Engel [1980]) without being able to present a rigorous proof of these results, or even to indicate how such a proof can be obtained.

Conclusions. In this short note we have only been able to mention a few of the many results available. For further information the reader should consult the lists of references in the papers already cited. A useful survey of higher-dimensional results can be found in Ryškov and Baranovskii [1976], and an extensive treatment of tiling problems in the Euclidean plane appears in Grünbaum and Shephard [1980a].

One must surely feel humble when one realizes that after two millennia of continuous effort by mathematicians, there are so many simple open problems and so much we do not know about the "familiar" three-dimensional space in which we live.

Acknowledgement. We are greatly indebted to all the colleagues who have suggested improvements of preliminary versions of this paper. The list of their names is too long to be included here; but we wish to make specific mention of the particularly great help we received from Peter Engel, Werner Fischer and Elke Koch. To Dr. Peter Engel we also thank for the excellent originals of Figures 10, 11 and 13.

\section{REFERENCES}

P. S. Aleksandrov (editor)

1969 Hilbert's Problems, Nauka, Moscow, 1969. (Russian) German translation: Die Hilbertschen Probleme, Akademische Verlagsgesellschaft Geest \& Portig, Leipzig, 1971. 
W. Baily

1908 On models of space-filling solids, Math. Gazette 4 (1908), 297-302.

J. Baracs

1979 Juxtapositions, Structural Topology 1 (1979), 59-72.

L. Baumgartner

1968 Zerlegung des vierdimensionalen Raumes in kongruente Fünfzelle, Math. Phys. Semesterber. 15 (1968), 76-86.

1971 Zerlegung des n-dimensionalen Raumes in kongruente Simplexe, Math. Nachr. 48 (1971), 213-224.

F. E. Browder (editor)

1976 Mathematical developments arising from Hilbert problems, Proc. Sympos. Pure Math., vol. 28, Amer. Math. Soc., Providence, R. I., 1976.

G. O. Brunner and F. Laves

1978 How many faces has the largest space-filling polyhedron? Zeitschr. Kristallographie 147 (1978), 39-43.

P. J. Cohen

1969 Decision procedures for real and p-adic fields, Comm. Pure Appl. Math. 22 (1969), 131-151.

H. S. M. Coxeter

1962 The classification of zonohedra by means of projective diagrams, J. Math. Pures Appl. 41 (1962), 137-156. Reprinted in: Twelve Geometric Essays, Southern Illinois Univ. Press, Carbondale, 1968, pp. 54-74.

1978 Review of "Three-dimensional nets and polyhedra" by A. F. Wells, Bull. Amer. Math. Soc. 84 (1978), 466-470.

K. Critchlow

1970 Order in space, Viking Press, New York, 1970.

L. Danzer

1968 Zerlegbarkeit endlichdimensionaler Räume in kongruente Simplices, Math. Phys. Semesterber. 15 (1968), p. 87.

H. E. Debrunner

1980 Pflasterungen des euklidischen Raumes mit kongruenten Simplexen (to appear).

B. N. Delone ( = Delaunay)

1932 Neue Darstellung der geometrischen Kristallographie, Zeitschr. Kristallographie 84 (1932), 109-149.

1961 A proof of the fundamental theorem of the theory of stereohedra, Dokl. Akad. Nauk. SSSR 138 (1961), 1270-1272. (Russian)

1969 On Hilbert's eighteenth problem, (Russian) pages 200-203 in Aleksandrov [1969]; pages 254-258 of the German translation.

B. N. Delone, N. P. Dolbilin and M. I. Stogrin

1978 Combinatorial and metric theory of planigons, Trudy Mat. In-ta AN SSSR 148 (1978), 109-140. (Russian)

B. N. Delone, R. V. Galiulin and M. I. Štogrin

1979 The contemporary theory of regular decompositions of the Euclidean space, pages 235-260 of Fedorov [1979]. (Russian)

B. Delone, N. Padurov and A. Aleksandrov

1934 Mathematical foundations of the structural analysis of crystals, ONTI, Moscow-Leningrad, 1934. (Russian)

B. N. Delone and N. N. Sandakova

1961 Theory of stereohedra, Trudy Mat. Inst. Steklov 64 (1961), 28-51. (Russian)

P. Engel

1980 Ueber Wirkungsbereichsteilungen mit kubischer Symmetrie, Zeitschr. Kristallographie (to appear).

E. S. Fedorov

1885 Foundations of the theory of figures, Acad. Sci. Peterburg 1885. Republished with comments, Akad. Nauk SSSR 1953. (Russian)

1899 Reguläre Plan-und Raumteilung, Abh. K. Bayer. Akad. Wiss. (II. Classe) 20 (1899), 465-588. Russian translation: Fedorov [1979]. 
1979 Regular partition of plane and space, (Russian; translation of Fedorov [1899]) Nauka, Leningrad, 1979.

W. Fischer

1979 Homogene Raumteilungen in konvexe Polyeder, Collected Abstracts, Symposium on

L. Föppl Mathematical Crystallography, Riederalp, Wallis (Switzerland), August 1979, page 5.

1914 Der Fundamentalbereich des Diamantgitters, Phys. Zeitschr. 15 (1914), 191-193.

M. Gardner

1975a On tessellating the plane with convex polygon tiles, Scientific American, July 1975, pp. 112-117.

1975b Mathematical games, Scientific American, December 1975, pp. 116-119.

1977 Extraordinary nomperiodic tiling that enriches the theory of tiles, Scientific American, January 1977, pp. 110-121.

J. Giles, Jr.

1979 Infinite-level replicating dissections of plane figures, J. Combinat. Theory A 26 (1979), 319-327.

M. Goldberg

1972 The space-filling pentahedra, J. Combinat. Theory A 13 (1972), 437-443.

1974a Three infinite families of tetrahedral space-fillers, J. Combinat. Theory A 16 (1974), 348-354.

1974b The space-filling pentahedra. II, J. Combinat. Theory A 17 (1974), 375-378.

1976 Several new space-filling polyhedra, Geometriae Dedicata 5 (1976), 517-523.

1977 On the space-filling hexahedra, Geometriae Dedicata 6 (1977), 99-108.

1978 On the space-filling heptahedra, Geometriae Dedicata 7 (1978), 175-184.

1979 Convex polyhedral space-fillers of more than twelve faces, Geometriae Dedicata 8 (1979), 491-500.

B. Grünbaum and G. C. Shephard

1977 The eighty-one types of isohedral tilings in the plane, Math. Proc. Cambridge Philos. Soc. 82 (1977), 177-196.

1978 Isohedral tilings of the plane by polygons, Comment. Math. Helv. 53 (1978), 542-571.

1980a Tilings and patterns, Freeman and Co., San Francisco, 1980 (to appear).

1980b Spherical tilings with transitivity properties, Proc. Coxeter Symposium (to appear).

G. Hajos

1942 Uber einfache und mehrfache Bedeckung des n-dimensionalen Raumes mit einem Würfelgitter, Math. Z. 47 (1942), 427-467.

E. Heesch, H. Heesch and J. Loef

1944 System einer Flächenteilung und seine Anwendung zum Werkstoff- und Arbeitsparen, Reichsminister für Rüstung und Kriegsproduktion, 1944.

\section{H. Heesch}

1934 Über Raumteilungen, Nachr. Gesellschaft Wiss. Göttingen, Neue Folge, 1 (1934), 35-42.

1935 Aufbau der Ebene aus kongruenten Bereichen, Nachr. Gesellschaft Wiss. Göttingen, Neue Folge, 1 (1935), 115-117.

1968a Reguläres Parkettierungsproblem, Westdeutscher Verlag, Köln-Opladen, 1968.

1968b Parkettierungsprobleme, Der Mathematikunterricht, Ernst Klett Verlag, Stuttgart, 1968, No. 4, pp. 5-45.

N. F. M. Henry and K. Lonsdale

1965 International Tables for X-Ray Crystallography, Vol. 1, Kynoch Press, Birmingham, 1965.

D. Hilbert

1900 Mathematische Probleme, Göttinger Nachrichten 1900, pp. 253-297; also Arch. Math. Phys. Ser. 3, 1 (1901), pp. 44-63 and 213-237. English translation: Mathematical prob-

H. Jansen lems, Bull. Amer. Math. Soc. 8 (1902), 437-479; reprinted in Browder [1976], pp. 1-34.

1909 Lückenlose Ausfüllung des $R_{n}$ mit gitterfömig angeordneten n-dimensionalen Quadern, Dissertation, Universität Kiel, Teubner, Leipzig 1909.

I. Kaplansky

1977 Hilbert's Problems (Preliminary edition), Lecture Notes in Mathematics, Univ. of Chicago, 1977. 
O.-H. Keller

1930 Über die lückenlose Erfüllung des Raumes mit Würfeln, J. Reine Angew. Math. 163 (1930), 231-248.

R. B. Kershner

1968 On paving the plane, Amer. Math. Monthly 75 (1968), 839-844.

E. Koch

1972 Wirkungsbereichspolyeder und Wirkungsbereichsteilungen zu kubischen Gitterkomplexen mit weniger als drei Freiheitsgraden, Ph. D. Thesis, Philipps-Universität Marburg/Lahn, 1972.

E. Koch and W. Fischer

1972 Wirkungsbereichstypen einer verzerrten Diamantkonfiguration mit Kugelpackungscharakter, Zeitschr. Kristallographic 135 (1972), 73-92.

\section{E. Lampe}

1911 Review of Baily [1908], Fortschritte Math. 39 (1911), p. 552.

J. Lawrence

1980 Tiling $R^{d}$ by translates of the orthants (to appear)

H. D. Löckenhoff and E. Hellner

1971 Die Wirkungsbereiche der invarianten kubischen Gitterkomplexe, Neues Jahrbuch für Mineralogie, Monatshefte. 1971, pp. 155-174.

B. B. Mandelbrot

1977 Fractals, Freeman and Co., San Francisco, 1977.

J. Milnor

1976 Hilbert's Problem 18: On crystallographic groups, fundamental domains, and on sphere packing, pp. 491-506 in Browder [1976].

H. Minkowski

1897 Allgemeine Lehrsätze über die konvexen Polyeder, Nachr. Gesellschaft Wiss. Göttingen, Math.-Phys. K1. 1987, pp. 198-219. Reprinted in: Gesammelte Abhandlungen, vol. 2, pp.

W. Nowacki 103-121. Teubner, Leipzig 1911; reprint Chelsea, New York, 1967.

1935 Homogene Raumteilung und Kristallstruktur, Ph. D. Thesis, E. T. H. Zürich, 1935.

1976 Über allgemeine Eigenschaften von Wirkungsbereichen, Zeitschr. Kristallographie 143 (1976), 360-368.

R. Penrose

1978 Pentaplexity: A class of non-periodic tilings of the plane, Eureka (Cambridge) 39 (1978), 16-22; reprinted in Math. Intelligencer 2 (1979), 32-37.

O. Perron

1940a Über lückenlose Ausfüllung des n-dimensionalen Raumes durch kongruente Würfel, Math. Z. 46 (1940), 1-26; 161-180.

1940b Modulartige lückenlose Ausfüllung des $R_{n}$ mit kongruenten Würfeln, Math. Ann. 117 (1940), 415-447.

1941 Modulartige lückenlose Ausfüllung des $R_{n}$ mit kongruenten Würfeln. II, Math. Ann. 117 (1941), 609-658.

K. Reinhardt

1918 Uber die Zerlegung der Ebene in Polygone, Ph. D. Thesis, Univ. Frankfurt a.M. Noske, Borna-Leipzig, 1918.

1928 Zur Zerlegung der euklidischen Räume in kongruente Polytope, S.-Ber. Preuss. Akad. Wiss. Berlin, 1928, pp. 150-155.

R. M. Robinson

1979 Multiple tilings of n-dimensional space by unit cubes, Math. Z. 166 (1979), 225-264.

1980. Can cubes avoid meeting face to face? The Mathematical Gardner, D. A. Klarner, ed. Prindle-Weber-Schmidt, Boston 1980 (to appear).

S. S. Ryškov and E. P. Baranovskii

1976 The C-types of $n$-dimensional lattices and primitive five-dimensional parallelohedra, Trudy Mat. Inst. Akad. Nauk SSSR 137 (1976). (Russian)

D. Schattschneider

1978 Tiling the plane with congruent pentagons, Math. Magazine 51 (1978), 29-44.

A. Seidenberg

1954 A new decision method for elementary algebra, Ann. of Math. 60 (1954), 365-374. 
K. Seitz

1975 Investigations in the Hajos-Redei theory of finite Abelian groups, DM 75-6. Department of Mathematics, Karl Marx University of Economics, Budapest, 1975. i + 45 pp., MR 53 \#655.

C. S. Smith

1978 Structural hierarchy in science, art, and history, On Aesthetics in Science, edited by J. Wechsler. The MIT Press, Cambridge, Mass., 1978, pp. 9-53.

F. W. Smith

1965 The structure of aggregates-a class of 20-faced space-filling polyhedra, Canad. J. Physics 43 (1965), 2052-2055.

S. K. Stein

1974 Algebraic tiling, Amer. Math. Monthly 81 (1974), 445-462.

M. I. Stogrin

1968 Regular Dirichlet partitions for the second triclinic group, Dokl. Akad. Nauk SSSR 183 (1968), 793-796. (Russian) English translation: Soviet Math. Dokl. 9 (1968), 1479-1482.

1973 Regular Dirichlet-Voronoi partitions for the second triclinic group, Trudy Mat. Inst. Steklov vol. 123 (1973). (Russian) English translation: Proc. Steklov Inst. Math. 123 Amer. Math. Soc., Providence, R. I., 1975.

A. V. Subnikov and V. A. Kopcik

1972 Symmetry in science and art, Nauka, Moscow 1972. (Russian) English translation: A. V. Shubnikov and V. A. Koptsik, Symmetry in Science and Art., Plenum Press, New York and London, 1974.

A. Tarski

1951 A decision method for elementary algebra and geometry, Univ. of California Press, Berkeley, Calif., 1951.

R. Williams

1972 Natural structure: Toward a form language, Eudaemon Press, Moorpark, Calif., 1972. Second edition, Dover, New York, 1979.

A. M. Zamorzaev

1965 On non-normal regular partitions of the Euclidean space, Dokl. Akad. Nauk SSSR 161 (1965), 30-31. (Russian)

Department of Mathematics, University of Washington, Seattle, Washington 98195

Department of Mathematics, University of EAst Angli, Norwich NR4 TTJ, England 
